\title{
The Impact of Online Opinion on Minimum Wage Policies on Firms' Innovation Performance
}

\author{
Shutter Zor \\ Accounting College, Wuhan Textile University, Wuhan 430200, Hubei Province, China \\ Shutter_Z@outlook.com
}

\begin{abstract}
Based on the realistic background of the accessibility of online public opinion texts, this paper selects tweets containing specific social security policy content from Sina Weibo during a total of ten years from 2011 to 2020 as the collection objects, uses the scrapy library in python to filter and crawl them to construct the online public opinion index, and evaluates the impact efficiency of the online public opinion index through data envelopment analysis (DEA). The empirical results show that the results of the DEA analysis of the online public opinion index differ somewhat between provinces and that the public opinion impact of this type of social security policy is statistically significant in favour of enhanced corporate innovation performance.
\end{abstract}

Keywords: Minimum wage, Online opinion analysis, Innovation performance, Data envelopment analysis.

\section{Introduction}

As deep learning and computer technology continue to improve and mature, algorithms have emerged that allow scholars to use non-quantitative, more informative text to uncover the depth of meaning behind it. Among the studies on formal texts, the integration of manually screened English dictionaries that are now widely recognised as being applicable to exploring the sentiment reflected in annual reports of listed companies dates back to 2011 when Loughran and McDonald [1] conducted manual screening. Unlike the simplicity of English, Chinese has ideational differences from English, and the same Chinese word can express many different meanings, which makes it very difficult to implement the extraction of Chinese emotion dictionaries. Likewise, some Chinese scholars have used manual discrimination to identify the emotions of a text, as the old saying goes, there are a thousand Hamlets in a thousand readers' minds. However, manual discrimination is not always correct, so this paper takes a machine learning approach to constructing an emotion lexicon and applies it to the calculation of the web emotion indicators in this paper.

In the above online public opinion lexicon, there are two differentiated emotions, positive and negative. Differentiated expressions of sentiment help public opinion departments to enhance their knowledge of online public opinion and provide an effective way to manage online public opinion, while also serving as a macro-regulatory tool for the government to intervene in the fairness of the market. China's law stipulates that the minimum wage can be considered differently according to the specific situation of each region and needs to be adjusted once every one to three years. The number of minimum wage adjustments by province and city from 2011 to 2020 is shown in Figure 1. As such a social security system is adjusted relatively frequently, it is inevitable that this will lead to changes in the direction of public opinion on the internet, and such changes are likely to cause some companies to sway from their own innovative decisions, resulting in a certain rational catering response This may lead to a certain amount of rational reaction, resulting in ineffective management. This paper uses a DEA approach to explain the changes in innovation performance of companies in different provinces, using the change in minimum wage as an explanatory variable.

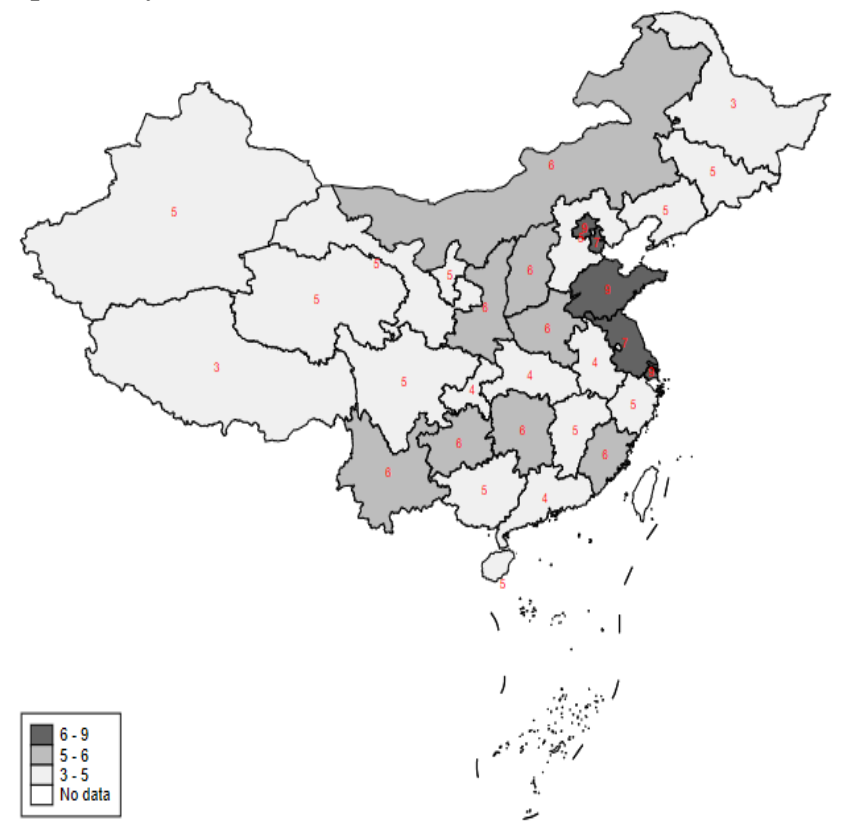

Figure 1: Frequency of minimum wage adjustments in major provincial administrative units in China over the past decade

\section{Variables}

For the measurement of mood indicators, this paper starts with a computational approach to extracting a mood lexicon. A machine learning penalty function for constructing an emotion lexicon with reference to the method of Yao et al. [2]

$$
\text { positive }=\frac{w_{n, N}}{\sum_{n} w_{n, N}} \times \frac{1}{1+\frac{w_{n, P}}{\sum_{n} w_{n, P}}}
$$

where $w_{n, N}$ is the number of occurrences of optional positive words $n$ in the set $N$ of annual reports with positive returns in the vocabulary of the annual report corpus from the Integrated Chinese Lexical Analysis System of the Chinese Academy of Sciences (ICTCLAS) and others. $w_{n, P}$ is the number of 
occurrences of the optional positive word $n$ in the set $N$ of annual reports with negative returns.

After obtaining a more objective and accurate sentiment lexicon, the text of the basic information in the annual reports of listed companies (basicmess) is intersected and filtered through Stata's text analysis function, with positive words (positive) added to the positive score and negative words (negative) added to the negative score, and finally aggregated into a comprehensive investor sentiment indicator, calculated as follows.

$$
\begin{aligned}
\text { positive }_{i t} & = \begin{cases}1, & \text { if positive } \cap \text { basicmess }=\text { true } ; \\
0, & \text { if positive } \cap \text { basicmess }=\text { false } .\end{cases} \\
\text { negative }_{i t} & = \begin{cases}1, & \text { if negative } \cap \text { basicmess }=\text { true } ; \\
0, & \text { if negative } \cap \text { basicmess }=\text { false } .\end{cases} \\
\text { OPOI }_{i t} & =\ln \left(\sum \text { positive }_{i t}-\sum \text { negative }_{i t}\right)
\end{aligned}
$$

where $O P O I_{i t}$ denotes the online opinion index of the minimum wage, with subscripts $i$ and $t$ representing different regions and years respectively. The definitions and symbols for the remaining indicators are shown in Table 1.

Table 1: Variable Symbol \& Definition

\begin{tabular}{c|c|c}
\hline Type & Symbol & Definition \\
\hline output & INNO & $\ln ($ Number of patent applications) \\
\hline \multirow{4}{*}{ input } & OPOI & Constructed according to the above method \\
\cline { 2 - 3 } & SIZE & $\ln$ (Total asset) \\
\cline { 2 - 3 } & RD & R\&D expenditure/operating income \\
\cline { 2 - 3 } & NATION & Percentage of state-owned shareholders \\
\cline { 2 - 3 } & BIG10 & Shareholding of top ten shareholders \\
\cline { 2 - 3 } & LEV & Total liabilities/Total assets \\
\cline { 2 - 3 } & ROA & Net Profit / Total Assets \\
\cline { 2 - 3 } & AGE & Number of years to establishment date \\
\hline
\end{tabular}

\section{Data Analysis}

\subsection{Basic Analysis}

The standard deviation of each variable is at an acceptable level and the data distribution is closer to a Gaussian distribution after a $95 \%$ tailing process for some of the extreme values, and the descriptive statistics for each variable are shown in Table 2.

Table 2: Variable Symbol \& Definition

\begin{tabular}{c|c|c|c|c|c}
\hline N=9246 & mean & sd & $\min$ & $\mathrm{p} 50$ & $\max$ \\
\hline INNO & 3.960 & 2.151 & 0.000 & 4.331 & 11.212 \\
\hline OPOI & 8.067 & 1.113 & 3.912 & 8.437 & 8.902 \\
\hline WAGE & 9.898 & 0.225 & 9.064 & 9.918 & 12.283 \\
\hline SIZE & 21.802 & 1.112 & 18.921 & 21.642 & 26.602 \\
\hline RD & 4.745 & 2.974 & 0.490 & 4.040 & 12.560 \\
\hline NATION & 0.094 & 0.136 & 0.000 & 0.000 & 1.000 \\
\hline BIG10 & 31.346 & 7.962 & 17.665 & 31.620 & 49.300 \\
\hline LEV & 0.358 & 0.177 & 0.089 & 0.343 & 0.688 \\
\hline ROA & 0.050 & 0.041 & -0.029 & 0.046 & 0.135 \\
\hline AGE & 16.019 & 5.001 & 7.000 & 16.000 & 25.000 \\
\hline
\end{tabular}

As can be seen from the table above, the water mean of INNO, the key variable of interest in this model, is 3.960 and the median is 4.331. Combining the minimum and maximum values, it is easy to see that the majority of companies have a low level of innovation performance, but there are very few manufacturing companies with outstanding capabilities. Among the characteristics of the variables, it is easy to see that the Internet opinion index is always more positive, i.e. the continuous increase in the minimum wage policy over the years has brought at least one unit more positive than negative sentiment to our Internet users. The results of the correlation analysis of the variables are shown in Figure 2.

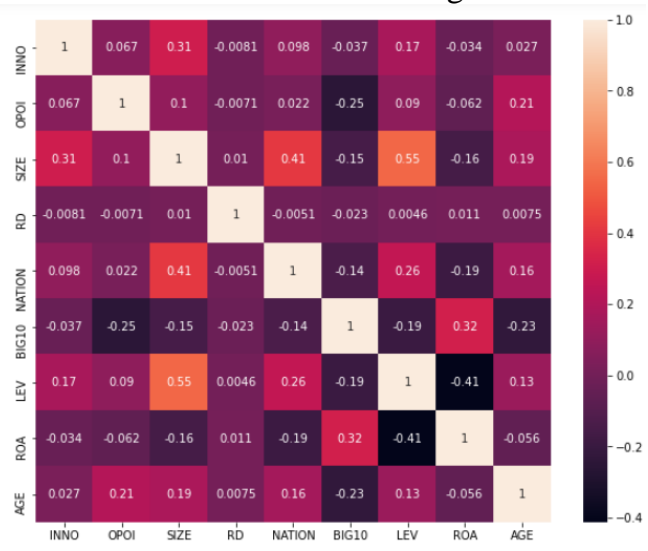

Figure 2: Pearson correlation coefficient

From the pearson correlation coefficient heat map, it is easy to find that most of the variables relevant to the study of this paper are correlated to some extent, and all of them are below 0.7 , which avoids the influence of multicollinearity to a certain extent, indicating that the selected variables are more reasonable.

\subsection{Data Envelopment Analysis}

Data envelopment analysis is a way of measuring sectoral technical efficiency using an operations research approach, and for the 25 main provinces in the sample data in this paper, with a total of eight input resources and one output outcome, then a combined input-output profile can be calculated by weighting.

$$
\begin{aligned}
\text { input }= & \alpha_{1} \mathrm{OPOI}+\alpha_{2} S I Z E+\alpha_{3} R D+\alpha_{4} N A T I O N \\
& +\alpha_{5} B I G 10+\alpha_{6} L E V+\alpha_{7} R O A+\alpha_{8} A G E \\
\text { output }= & \text { INNO }
\end{aligned}
$$

Where $\alpha_{1}-\alpha_{8}$ denotes the corresponding weight of the input resources. Then, in such a case, for each decision unit (province, denoted by PROV), the output-input ratio can be expressed as:

$$
P_{R O V}=\frac{\text { output }_{i t}}{\text { input }_{i t}}
$$

Based on the above inferences, this paper calculates a DEA model based on input-oriented constant radial payoffs of scale for the period 2011-2020 for comparing innovation performance outputs driven by decision variables such as online public opinion in recent years between different provinces, as shown in Table 3.

Table 3: DEA results for selected provinces and municipalities

\begin{tabular}{c|c|c|c|c}
\hline \multirow{2}{*}{ PROV } & \multicolumn{4}{|c}{ YEAR } \\
\cline { 2 - 5 } & 2014 & 2016 & 2018 & 2020 \\
\hline Beijing & 1.000 & 0.975 & 1.000 & 1.000 \\
\hline Shanghai & 0.855 & 0.928 & 0.971 & 1.000 \\
\hline Hubei & 0.810 & 0.929 & 1.000 & 1.000 \\
\hline
\end{tabular}

Notes: *The rest of the provincial data can be requested from the author by email.

From the table above, it is easy to see that technical efficiency 
rises in most provinces and municipalities between 2011 and 2020 , and that nearly half of the provinces show technical efficiency by 2020 , i.e. the output of innovation performance resulting from the influence of online public opinion is very efficient for companies domiciled in these areas, and the minimum wage is raised more frequently and by larger amounts in these provinces in relative terms. Other provinces, particularly those with relatively poor economic conditions, are less efficient in terms of input and output. This suggests that the impact of online public opinion triggered by the occurrence of minimum wage variables in different provinces and municipalities is inconsistent, promoting innovation in some provinces and struggling to have a greater effect on innovation in others.

\section{Discussion and Recommendations}

The text was crawled using the more mature scrapy for Sina Weibo text data, and a server was set up for web crawling at 15 second intervals to ensure continuity and data integrity. In order to exclude the influence of some recruitment information, the topic of "basic wage" was excluded from the text in order to get the original text about the sentiment of minimum wage. The text was filtered through a previously trained sentiment lexicon to obtain positive and negative sentiment indices for the text, which were integrated into an online opinion index for subsequent empirical research. Using tweets containing "minimum wage" on Sina Weibo between 2011 and 2020 as a sample, the online opinion text index was constructed to analyse the impact of changes in online opinion on minimum wage on firms' innovation performance in both grounded theory and statistical sense, and to analyse the impact of such changes on the innovation performance of firms in the main provinces and municipalities in the sample. It also analyses the changes in technical efficiency caused by such changes in the main provinces of the sample. The paper finds that provinces and municipalities with frequent minimum wage changes and better welfare benefits have higher output efficiency in firm innovation performance than those with relatively less frequent minimum wage changes. However, there are limitations to the paper, namely the technical means to analyse the textual information more precisely.

\section{Acknowledgement}

This research was funded by Wuhan Textile University Postgraduate Innovation Fund Project, grant number 2015153010 .

\section{Data Availability Statement}

All data in this paper are from CSMAR, except for the sentiment indicator data, which were constructed by the authors. If interested in more information about this article, the original data and code can be requested from the author.

\section{References}

[1] Loughran T, Mcdonald B. When is a liability not a liability? textual analysis, dictionaries, and 10-Ks [J]. The Journal of Finance, 2011, 66(1).
[2] Yao et al. Tone, sentiment and market impact: Based on the financial sentiment dictionary [J]. Journal of Management Sciences in China, 2021, 24(05): 26-46.

[3] Hanxu Zhao. Does Big4 audit improve the quality of corporate information, an empirical analysis based on PSM [J]. Journal of Innovation and Social Science Research (JISSR), 2021, 08(09): 15.

\section{Author Profile}

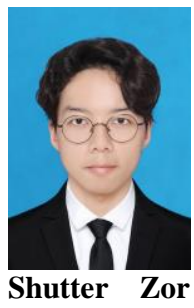

(ORCID: 0000-0003-1286-4765) received his Bachelor's degree in Management from Wuhan Textile University in 2020 and enrolled in a Master's degree in Accounting at Wuhan Textile University in the same year. Expert in the use of scientific tools such as Stata, Python and Matlab, with an interest in quantitative methods of analysis. His main research interests are technological innovation and he is open to international cooperation. 